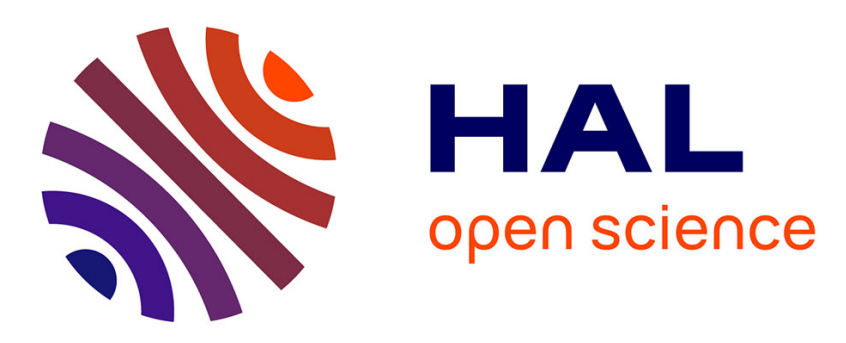

\title{
Effective permittivity of 3D lossy dielectric composite materials
}

Philippe Clauzon, Laurent Krähenbühl, Alain Nicolas

\section{To cite this version:}

Philippe Clauzon, Laurent Krähenbühl, Alain Nicolas. Effective permittivity of 3D lossy dielectric composite materials. IEEE Transactions on Magnetics, 1999, 35 (3 Part 1), pp.1223-1226. $10.1109 / 20.767170$. hal-00141538

\section{HAL Id: hal-00141538 \\ https://hal.science/hal-00141538}

Submitted on 20 Apr 2007

HAL is a multi-disciplinary open access archive for the deposit and dissemination of scientific research documents, whether they are published or not. The documents may come from teaching and research institutions in France or abroad, or from public or private research centers.
L'archive ouverte pluridisciplinaire HAL, est destinée au dépôt et à la diffusion de documents scientifiques de niveau recherche, publiés ou non, émanant des établissements d'enseignement et de recherche français ou étrangers, des laboratoires publics ou privés. 


\title{
Effective Permittivity of 3D Lossy Dielectric Composite Materials
}

\author{
P. Clauzon, L. Krähenbühl, A. Nicolas \\ Ecole Centrale de Lyon, CEGELY. , UPRRSA C.N.R.S. 5005, BP 163, 69131 Ecully Cedex, France \\ sis.

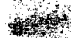

Abstract -In order to better know the behavior of dielectric composite materials, we employ a Boundary Integral Equations Method to calculate both the complex effective permittivity and the effective loss tangent of $3 \mathrm{D}$ lossy dielectric composite materials. The topological arrangements considered are periodic lattices of inclusions. A method for the exploitation of the results has been proposed.

Index terms -dielectric materials, nonhomogeneous media, dielectric losses, boundary integral equations.

\section{INTRODUCTION}

The materials considered in this survey are a mixture of two dielectric media. The elementary cell geometries of the materials are simulated with the help of a modified 3D C.A.D. package (PHI3D).

The aim of this survey is to calculate the complex effective permittivity and the effective loss tangent of these materials, this for different values of permittivities and volume fractions of the inclusions.

Thereafter, some techniques of interpolation in two dimensions were used in order to calculate the complex effective permittivity and the loss tangent of the dielectric composite materials from the previous results.

\section{STUDIED STRUCTURES}

We considered two types of dielectric composite materials. For the both materials, the matrix is a dielectric having the properties of the air. The inclusions are a same dielectric of which the real part of the relative permittivity varies from 3 to 30 . The permittivity of the inclusions is complex because they have lossy dielectric characteristics. The loss tangent of each inclusion is equal to 0.01 . The differences between the two materials are the shapes of the matrix and the inclusions. The first material has spherical inclusions arranged in a cubic lattice (see Fig.1) whereas the second one has ellipsoidal inclusions arranged periodically in the three spatial directions (see Fig.2).

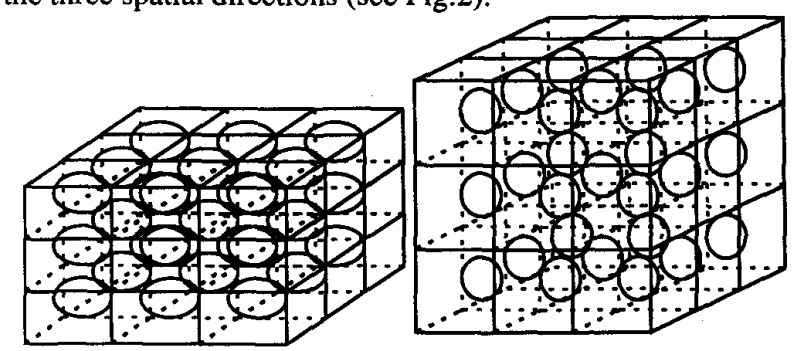

Fig. 1. Geometry of the material with ellipsoid inclusions..

Fig. 2. Geometry of the material with spherical inclusions.

Manuscript received June 3, 1998.

P. Clauzon, pclauzon@eea.ec-lyon.fr; A. Nicolas, 33472186096 , fax 33478433717 Alain.Nicolas@ec-lyon.fr; http://cegely.ec-lyon.fr.

\section{A. Elementary cell characteristics}

As we will see it later, the calculations are achieved by Boundary Integral Equations. The heaviness of the computations is reduced considering only one elementary cell [1], because of the periodicity of the materials.

The elementary cell of the material with ellipsoidal inclusions, is gotten from the cell of the material with spherical inclusions, by homothetical transformation of length ratio $\mathrm{k}=2$ according to the $z$ axis, and of center $O$, center of the cell (see Fig. 3.). The height $h$ is therefore equal to $4 \mathrm{~mm}$, $l=L=2 \mathrm{~mm}$ and $b=c=a / 2$. When the ellipsoid is not in contact with the limits of the matrix, that is to say for $a<2$, the volume fraction takes the following expression:

$$
f=\frac{4 \pi a b c}{3 L l h}
$$

When the ellipsoid intercepts the sides of the parallelepiped [2], the analytic expression of the volume fraction is hard to find. Its calculation is however possible. In fact, homothetical transformation preserves the forms of the volumes, but enlarges them of $\mathrm{k}^{3}$ ratio. The volume fraction being a volume ratio, this one remains unchanged compared with the material with spherical inclusions.

The material with spherical inclusions has an elementary cell (see Fig. 4.) of which inclusion radius $R$ varies from 0.457 $\mathrm{mm}$ to $1.41 \mathrm{~mm}$. The cubic matrix presents an edge $a$ of 2 $\mathrm{mm}$ in length.

The volume fraction is:

$$
f=\frac{\pi R^{3}}{6} \text { with } R \leq 1
$$

and $f=\frac{\pi R^{3}}{6}-\frac{\pi}{4}(R-1)^{2}(2 R-1)$ with $1 \leq R \leq \sqrt{2}$.

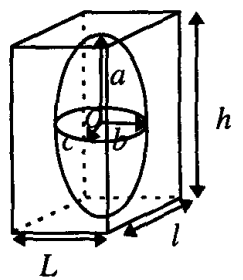

Fig. 3. Geometry of an elementary cell of the material with ellipsoidal inclusions.

\section{B. Boundary conditions}

During simulations, the boundary conditions for each geometry are set in the following way: 
a)

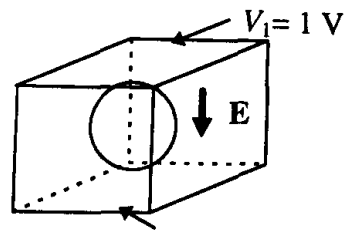

b)

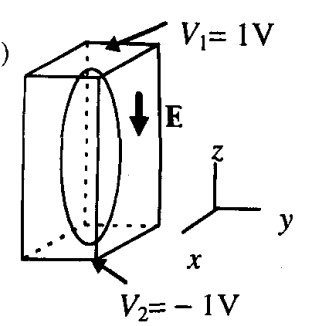

c)

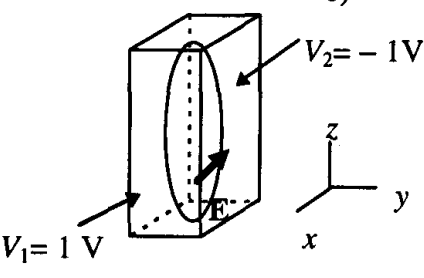

Fig. 4. Boundary conditions for a) the material with spherical inclusions, b) the material with ellipsoidal inclusion in the $\mathrm{z}$ direction and $\mathrm{c}$ ) in the $\mathrm{x}$ direction.

All the surfaces not including information are set with some homogeneous Neumann conditions $\partial V / \partial n=0$.

\section{NUMERICAL METHOD}

The permittivity of the studied materials is complex since their inclusions present dielectric losses [3]. The effective permittivity of the material with spherical inclusions takes merely the following shape:

$$
\underline{\varepsilon}=\varepsilon^{\prime}-j \varepsilon^{\prime \prime} \text { and } \tan \delta=\varepsilon^{\prime \prime} / \varepsilon^{\prime},
$$

since the material is isotropic

On the other hand, the effective permittivity of the material with ellipsoidal inclusions must be calculated in the $z$ direction and in the $\mathrm{x}$ or $\mathrm{y}$ direction, as this material is anisotropic:

$$
\begin{gathered}
\underline{\varepsilon}_{z}=\varepsilon_{z}^{\prime}-j \varepsilon_{z}^{\prime \prime}, \tan \delta_{z}=\frac{\varepsilon_{z}^{\prime \prime}}{\varepsilon_{z}^{\prime}}, \\
\text { and } \\
\underline{\varepsilon}_{x \text { ow } y}=\varepsilon_{x \text { ou } y}^{\prime}-j \varepsilon_{x \text { ou } y}^{\prime \prime}, \tan \delta_{x \text { or } y}=\frac{\varepsilon_{x o r y}^{\prime \prime}}{\varepsilon_{x \text { or } y}^{\prime}} .
\end{gathered}
$$

The dimensions of the inclusions remain lower than the wavelength of the applied field whichever the used frequency $(F \ll v / 2 R, v$ is the speed of propagation of the applied field, here the highest frequency is $1 \mathrm{MHz}$ ). We will consider that there is no propagation. Moreover, the imaginary part of the permittivity of the inclusions as well as the loss tangent, are small compared to the real part of this permittivity. The displacement currents are negligible and have no influence on the electric field ( the frequency must be above the microwaves frequencies: $300 \mathrm{MHz}$ ). One consider that the electric potential $V$ is sinusoidal with a $\mathrm{F}$ frequency and has an amplitude equal to the static value. The dissipated power is therefore equal to:

$$
P_{\text {dissipated }}=\sum_{i=1}^{\text {Nunber of lossy media }} \frac{1}{2} \iiint_{v} \varepsilon_{i} E^{2} \tan \delta_{i} 2 \pi F d x d y d z
$$

The dissipated power correspond to the dielectric losses. Each loss tangent $\tan \delta_{i}$ is identical in all the corresponding lossy medium $i$.

All the previous hypotheses lead us to solve a static problem, that is to say the Laplace's equation. This equation is solved with the help of the PHI3D software based upon the Boundary Integral Equations Method [4]. The electric potential $V$ is determined on the whole volume domain $v$ with the aid of (7),

$$
\text { c. } V(p)=-\iint_{s}\left(V \frac{\partial G}{\partial n}-G \frac{\partial V}{\partial n}\right) d x d y,
$$

where $G$ is the Green's function, $c$ is the solid angle under which the point $p$ sees the volume $v, \mathrm{n}$ stands for the normal direction, and $S$ is the bounding surface of the domain $v$.

With this numeric method, only the surfaces bounding the volumes are meshed. The computation of the integral above in all points of the mesh grid leads us to solve a linear system. This system is construct from physical properties on the boundaries and from boundary conditions. The results of this system, $V$ and $\partial V / \partial n$ are gotten in each point of the mesh grid. Only $1 / 8^{\text {th }}$ of the geometry is simulated because the system presents two symmetries and one anti-symmetry. This reduction of the studied domain allows to reduce the time of computation and the space memory taken by the matrices of the system.

In order to determine the effective complex permittivity of the materials, the real and imaginary parts, and the effective loss tangent, an electric field is set in a direction of the $x y z$ coordinate system, by fixing the potential $V$ on the perpendicular surfaces to the direction of the field. For the material with spherical inclusions, only one of the three directions of the xyz-coordinate system will be necessary since the material is isotropic. On the other hand, for the material with ellipsoidal inclusions, the computations will be achieved for the direction of the $x y z$-coordinate system parallel to the long axis of the ellipsoid and for one of the two perpendicular directions, this material is anisotropic.

The real part of the effective permittivity of each material is calculated from the flux of the electric induction $\mathbf{D}$ through one of the perpendicular surfaces to the direction of the applied field:

$$
\phi_{z}=\iint_{s} \mathbf{D}_{z} \cdot \mathbf{n} d x d y=\varepsilon_{z}^{\prime}|\mathbf{E}| S=\varepsilon_{z}^{\prime} \frac{V_{1}-V_{2}}{e} S, \text { for the } z
$$

direction for example.

$$
\varepsilon_{z}^{\prime}=\frac{\Phi_{z} e}{\left(V_{1}-V_{2}\right) S}
$$

with $S$ area of the considered surface, $\varepsilon_{z}^{\prime}$ is the real part of the effective permittivity in the $z$ direction, $\phi_{\mathrm{z}}$ being the flux of the electric induction $\mathbf{D}$ in the $z$ direction, $e$ is the distance between the two perpendicular surfaces to the field direction (see Fig. 2-3-4 for the significance of the different variables no specified).

The imaginary part of the effective permittivity of each material is computed from the dielectric losses. It is a matter therefore of calculating the volume integral of the expression 
(7) numerically. In order to achieve this calculation, one comes back to the Maxwell's equations:

By integrating (10),

$$
\nabla(-\varepsilon \nabla V)=0
$$

$$
\iiint_{V} \varphi \nabla(-\varepsilon \nabla V) d x d y d z=0
$$

this whichever the $\varphi$ weighed function belonging to $v$. By application of the first identity of Green, we have:

$$
\iiint_{v} \varepsilon^{\prime} \mathbf{E}^{2} d x d y d z=\oiint_{s} V \varepsilon_{0} \varepsilon_{r}^{\prime} \frac{\partial V}{\partial n} d x d y
$$

The terms $V$ and $\varepsilon_{r}^{\prime} \partial V / \partial n$ are the results of the boundary integral formulation used in PHI3D. By modifying PHI3D to calculate the flux of the expression $V \varepsilon_{r}^{\prime} \partial V / \partial n$ through the surfaces bounding the lossy materials and for which $\partial V / \partial n$ is different of zero, we compute the dielectric losses with the help of expression (7). The knowledge of the dielectric losses leads us to the imaginary part of the effective permittivity:

$$
\begin{gathered}
\text { Dielectric losses }=\frac{1}{2} \varepsilon_{r}^{\prime \prime} \varepsilon_{0} \frac{S}{e} \omega\left(V_{1}-V_{2}\right)^{2} \\
\varepsilon_{r}^{\prime \prime}=\frac{2 \times \text { Dielectric losses } \times e}{S \varepsilon_{0} \omega\left(V_{1}-V_{2}\right)^{2}} \\
\tan \delta_{\text {efsective }}=\frac{\varepsilon_{r}^{\prime \prime}}{\varepsilon_{r}^{\prime}}
\end{gathered}
$$

The results, the calculation of the real and imaginary parts of the effective permittivity as well as the effective loss tangent, are represented, for each material, as a function of the volume fraction and of the real part of the permittivity of the inclusions. A representation as a function of the frequency is not proposed because if one observes the formulation, the frequency does not appear after simplification. A representation as a function of the frequency would give therefore only a constant curve.

For the first material with ellipsoidal inclusions, we represented the results successively for the $z$ direction, then for the perpendicular direction $x$ or $y$.

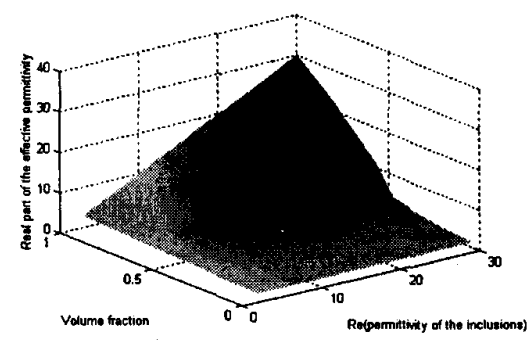

Fig. 7. Real part of the effective permittivity of the material with ellipsoidal inclusions, according to the $z$ direction.

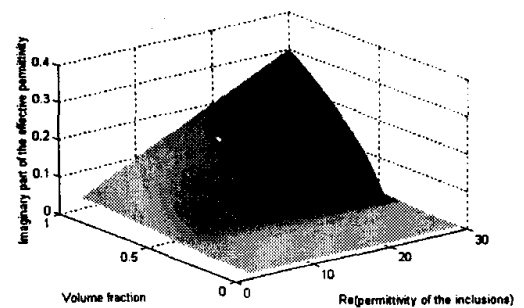

Fig. 8. Imaginary part of the effective permittivity of the material with ellipsoidal inclusions, according to the $z$ direction.

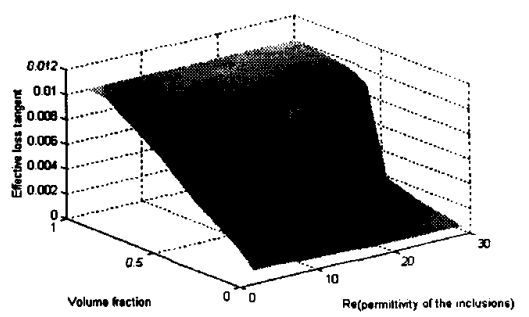

Fig. 9. Effective loss tangent of the material with ellipsoidal inclusions, according to the $z$ direction.

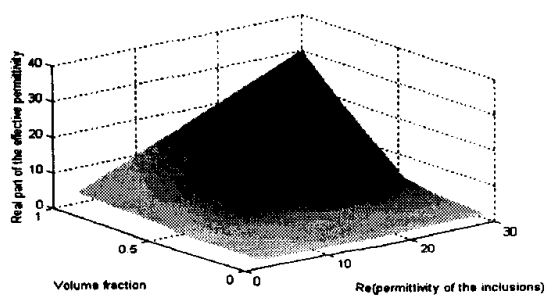

Fig. 10. Real part of the effective permittivity of the material with ellipsoidal inclusions, according to the $x$ or $y$ direction.

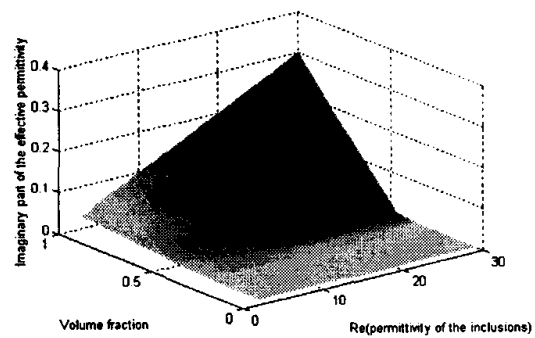

Fig. 11. Imaginary part of the effective permittivity of the material with ellipsoidal inclusions, according to the $x$ or $y$ direction.

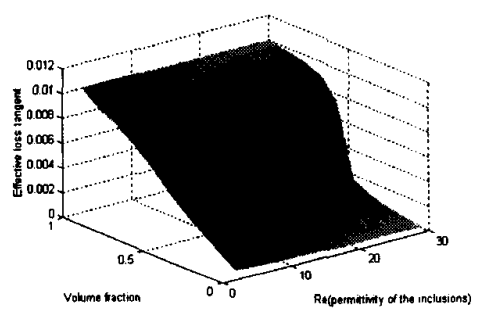

Fig. 12. Effective loss tangent of the material with ellipsoidal inclusions, according to the $x$ or $y$ direction. 
For this material with ellipsoidal inclusions, one notes that the real and imaginary parts evolve progressively as a function of the volume fraction, according to a polynomial form, with a marked increase of this evolution, shortly before the fraction 0.5 and beyond. The evolution of the effective permittivity, real and imaginary parts, is almost linear as a function of the real part of the permittivity of the inclusions. The loss tangent present two different regions as a function of the volume fraction, a first region where the loss tangent remains weak and close to the lowest fraction, a second region where this tangent is important and near to the inclusion loss tangent. This division in two regions brings to the material a certain selectivity to its properties. All these remarks are valid both for the results in the $z$ direction and in the perpendicular direction. For the two directions, the shapes of the curves look alike, but if one could superimpose these curves, one would note that the values in the $z$ direction, are more important, except for upper limit values, and shows therefore that the material possesses a privileged direction, the $z$ direction.

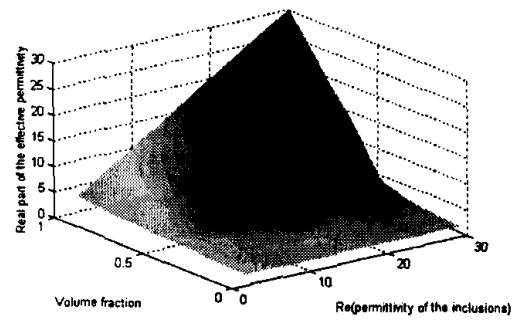

Fig. 13. Real part of the effective permittivity of the material with spherical inclusions, according to the $z$ direction.

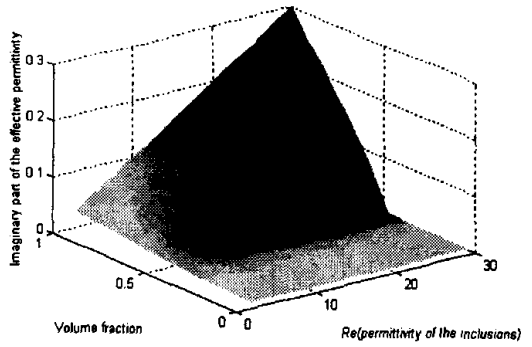

Fig. 14. Imaginary part of the effective permittivity of the material with spherical inclusions, according to the $z$ direction.

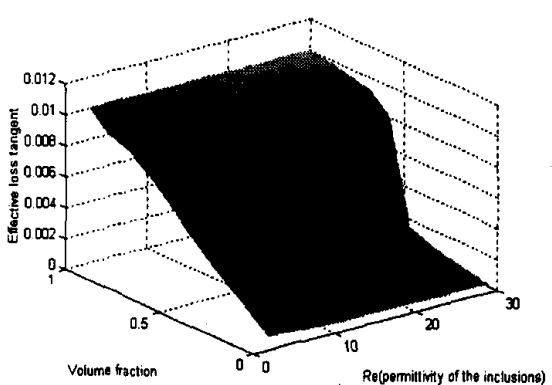

Fig. 15. Effective loss tangent of the material with spherical inclusions, according to the $z$ direction.
All the observations made for the material with ellipsoidal inclusions are valid for this material, with the exception of the direction of calculations. This material, due to the geometry of its inclusions, is isotropic. The computations are achieved in one of the directions of the coordinate system, and are therefore valid in the two others directions.

\section{EXPLOITATION OF THE RESULTS}

So that the previous results are useful, we used two methods of interpolation in two dimensions, in order to compute the effective complex permittivity in some unknown points of the data bank, which are in the limits of the results, but which are not part of the results. These methods will be used to know the properties of materials with similar structures to those of this present survey, but with different proportions between inclusions and matrices, for example before their conception.

In the same way, this kind of methods allows us to represent these heterogeneous materials with some homogeneous equivalent ones, always for some materials having similar structures to those of the survey. So a finite element mesh would required few elements, which is not the case when one studies a composite material with small and numerous inclusions. We will not present the two methods of interpolation here. The interested readers will see [5] [6]. The first method of interpolation comes from the MATLAB software. This method has been used for the first steps of the development of the exploitation method. Finally, we chose a second method based on a spline bicubic interpolation routine, written in $\mathrm{C}$, easily portable on different computers.

\section{CONCLUSION}

With modifications and approximations, the BIE method can be useful to compute the complex effective permittivity and the effective loss tangent of 3D dielectric composite materials. A method of exploitation of these results has been proposed with the view of creating a data bank and of introducing this data in a field calculation package.

\section{REFERENCES}

[1] P. K. Ghosh, M.E. Azimi, "Numerical calculation of effective permittivity of lossless dielectric mixture using Boundary Integral Method", IEEE Trans. on Diel. and Elect. Insul., vol. 1, no. 6, pp. 975 981, 1994.

[2] L. C. Shen, C. Liu, J. Korringa, K. J. Dunn, "Computation of conductivity and dielectric constant of periodic porous media", J. Appl. Phys. 67 (11), pp.7071-7081, june 1990.

[3] B. Sareni, L. Kränhenbühl, A. Beroual and C. Brosseau, "Complex effective permittivity of a lossy composite material", J. Appl. Phys. 80 (8), pp.4560-4565, october 1996.

[4] C. A. Brebbia, The Bondary Element Method for Engineers, London Pentech Press, 1980, pp. 1-103.

[5] P. Clauzon, A. Nicolas, " Effective permittivity of a dielectric composite material as a function of two parameters", 4th Internatioal Workshop on Electric and Magnetic Fields, Marseille(France), 12-15 may 1998, pp. 463-468.

[6] W. H. Press, S. A. Teukolsky, W. T. Vetterling, B. P. Flannery, NUMERICAL RECIPES IN C The art of scientific computing, 2nd ed., Cambridge U.S.A. : Cambridge University Press, 1992, pp. 105-128. 